\title{
A Note on the Discrete Spectrum of Gaussian Wells (I): The Ground State Energy in One Dimension
}

\author{
G. Muchatibaya, ${ }^{1}$ S. Fassari, ${ }^{2,3}$ F. Rinaldi, ${ }^{2,3}$ and J. Mushanyu ${ }^{1}$ \\ ${ }^{1}$ Department of Mathematics, University of Zimbabwe, P.O. Box MP167, Mount Pleasant, Harare, Zimbabwe \\ ${ }^{2}$ Universitá Degli Studi Guglielmo Marconi, Via Plinio 44, 00193 Rome, Italy \\ ${ }^{3}$ CERFIM, P.O. Box 1132, CH-6601 Locarno, Switzerland
}

Correspondence should be addressed to G. Muchatibaya; giftmuchatibaya@gmail.com

Received 15 January 2016; Accepted 7 February 2016

Academic Editor: Pavel Kurasov

Copyright (C) 2016 G. Muchatibaya et al. This is an open access article distributed under the Creative Commons Attribution License, which permits unrestricted use, distribution, and reproduction in any medium, provided the original work is properly cited.

The ground state energy $E_{0}(\lambda)$ of $H_{\lambda}=-d^{2} / d x^{2}-\lambda e^{-x^{2}}$ is computed for small values of $\lambda$ by means of an approximation of an integral operator in momentum space. Such an approximation leads to a transcendental equation for which $\epsilon_{0}(\lambda)=\left|E_{0}(\lambda)\right|^{1 / 2}$ is the root.

\section{Introduction}

In this brief note, we are concerned with the calculation of the ground state energy of the Hamiltonian:

$$
H_{\lambda}=-\frac{d^{2}}{d x^{2}}-\lambda e^{-x^{2}}
$$

with the latter being regarded as a self-adjoint operator on the domain $H^{2,2}(-\infty, \infty)$, that is, the second Sobolev space. Its form domain $Q\left(H_{\lambda}\right)$ is obviously the first Sobolev space.

Although the general features of the spectrum of our Hamiltonian are not different from those of the well-known rectangular well, we can take advantage of some special features of the Gaussian attractive potential to determine the ground state of $H_{\lambda}$ with great accuracy when $\lambda$ is small.

From the point of view of possible applications, the spectroscopy of such a potential might be of interest in relation to models of "artificial atoms" in the growing field of nanotechnology. For example, in a review article [1], a model of an artificial atom is given by using the two-dimensional harmonic oscillator potential. Although the latter provides a good approximation for the lowest eigenenergies, it is not exactly what we should expect of an atom, due to the absence of the absolutely continuous spectrum. It is therefore interesting to investigate the spectrum of Gaussian wells, since the latter potentials have the typical properties of shortrange potentials but also those of the harmonic oscillator near the bottom of the well.

\section{Calculation of the Ground State Energy}

As is well known to the mathematical physics community, the Birman-Schwinger kernel is a very useful tool to study the bound states of one-dimensional Hamiltonians with potentials belonging to $S_{d}=L^{1}\left(-\infty, \infty ;(1+|x|)^{d} d x\right), d=$ 1,2 (see [2-6]), and also, recently, the work of Fernández $[7,8]$. Used in combination with the Fredholm determinant or similar perturbative arguments, the B-S kernel leads to the well-known approximation for the ground state energy as a function of the coupling constant $\lambda$ :

$$
\begin{aligned}
\epsilon_{0}(\lambda)= & \left|E_{0}(\lambda)\right|^{1 / 2} \\
= & \frac{\lambda\|V\|_{1}}{2}-\frac{\lambda^{2}}{2\|V\|_{1}} \iint V(x)|x-y| V(y) d x d y \\
& +o\left(\lambda^{2}\right)
\end{aligned}
$$

for the rather general case $-d^{2} / d x^{2}-\lambda V, V(x) \geq 0, V \in S_{1}$.

We would like to point out that a similar expansion can also be obtained by means of the so-called Titchmarsh-Weyl 
$m$-function [9-11]. In our specific case, it is clear that, due to the exponential decay of the Gaussian, we could continue the expansion without limit to get the Taylor series for $\epsilon_{0}(\lambda)$. However, that is not what we are going to do in the following, since we are rather interested in an approximation that could give a better evaluation using a smaller number of terms.

Let us check how accurate (2) is in our particular case for a certain value of the coupling constant. Setting $\lambda=0.1$ and omitting the error term, we get, after calculating exactly the double integral,

$$
\left|E_{0}(\lambda)\right|^{1 / 2}=0.05 \sqrt{\pi}-\frac{0.005 \sqrt{2 \pi}}{\sqrt{\pi}}
$$

leading to

$$
E_{0}(0.1) \cong-6.65 \times 10^{-3}
$$

It is important to note that the $\lambda^{2}$-correction term $0.005 \sqrt{2}$ is relatively large, roughly, $7 \times 10^{-3}$, which is approximately $8 \%$ of the first term $0.05 \sqrt{\pi}$ in the expansion. This shows that, even for a relatively small value of the coupling constant, it is necessary to include the $\lambda^{2}$-correction term to get a good evaluation. Hence, as soon as $\lambda$ increases, more and more terms are required to get a correct evaluation.

By using an alternative technique, however, we have found out that it is possible to compute the ground state energy with greater accuracy by determining the root of a transcendental equation.

The crucial step is to use the trace class operator $B(\epsilon)$ with integral kernel in momentum space given by

$$
B\left(p, p^{\prime} ; \epsilon\right)=\left(p^{2}+\epsilon^{2}\right)^{-1 / 2} \frac{\widehat{V}\left(p-p^{\prime}\right)}{(2 \pi)^{1 / 2}}\left(p^{\prime 2}+\epsilon^{2}\right)^{-1 / 2}
$$

in place of the corresponding B-S kernel, following the ideas used in [12].

After computing the Fourier transform of our specific potential, the kernel can be rewritten as

$$
B\left(p, p^{\prime} ; \epsilon\right)=\frac{1}{2 \sqrt{\pi}} \frac{e^{-p^{2} / 4}}{\left(p^{2}+\epsilon^{2}\right)^{1 / 2}} e^{p p^{\prime} / 2} \frac{e^{-p^{\prime 2} / 4}}{\left(p^{\prime 2}+\epsilon^{2}\right)^{1 / 2}} .
$$

Using the Taylor expansion of the central exponential, our trace class operator can be rewritten as an infinite sum of rank-one operators; namely,

$$
B(\epsilon)=\frac{1}{2 \sqrt{\pi}} \sum_{n=0}^{\infty} \frac{1}{2^{l} l !}\left|f_{l}(\epsilon)\right\rangle\left\langle f_{l}(\epsilon)\right|
$$

where

$$
f_{l}(p ; \epsilon)=\frac{p^{l} e^{-p^{2} / 4}}{\left(p^{2}+\epsilon^{2}\right)^{1 / 2}} .
$$

Obviously,

$$
\|B(\epsilon)\|_{1}=\frac{1}{2 \sqrt{\pi}} \int_{-\infty}^{\infty} \frac{d p}{p^{2}+\epsilon^{2}}=\frac{\sqrt{\pi}}{2 \epsilon}
$$

using a well-known result for the explicit calculation of the trace class norm of positive integral operators with continuous kernels (see [13], page 65). Of course, apart from the two subsets of even- and odd-labeled rank-one operators, the operators in the series are not mutually orthogonal. However, it is not difficult, at least conceptually, to transform the sum of all the even-labeled (odd-labeled, resp.) rankone operators into an infinite sum of mutually orthogonal projectors and a sum of nilpotent rank-one operators by constructing an orthogonal system for the functions $f_{l}(p ; \epsilon)$. For example, the function $f_{2}(p ; \epsilon)=p^{2} e^{-p^{2} / 4} /\left(p^{2}+\epsilon^{2}\right)^{1 / 2}$ can be expressed as

$$
\alpha_{2}(\epsilon) \frac{e^{-p^{2} / 4}}{\left(p^{2}+\epsilon^{2}\right)^{1 / 2}}+\frac{\left[p^{2}-\alpha_{2}(\epsilon)\right] e^{-p^{2} / 4}}{\left(p^{2}+\epsilon^{2}\right)^{1 / 2}}
$$

where

$$
\alpha_{2}(\epsilon)=\frac{(2 \pi)^{1 / 2}}{\left\|f_{0}(\epsilon)\right\|_{2}^{2}}-\epsilon^{2}
$$

It is immediate to check that the second summand in (10) is orthogonal to the first.

If we are interested in the case of small $\lambda$ 's, for which the ground state is the only bound state, a fairly good evaluation of the ground state energy can be obtained by neglecting all the nilpotent operators and taking only the first rankone operator $\left|f_{0}(\epsilon)\right\rangle\left\langle f_{0}(\epsilon)\right|$ among the diagonal rank-one operators, since the norm of $f_{0}(\epsilon)$ is the only one that diverges as $\epsilon \rightarrow 0_{+}$. Then, in place of the correct equation $\operatorname{det}(I-$ $\lambda B(\epsilon))=0$ determining the eigenvalues $\epsilon_{l}(\lambda)$, we can use the much simpler equation:

$$
\operatorname{det}\left[I-\frac{\lambda}{2 \sqrt{\pi}}\left|f_{0}(\epsilon)\right\rangle\left\langle f_{0}(\epsilon)\right|[1-\lambda M(\epsilon)]^{-1}\right]=0,
$$

where $M(\epsilon)=B(\epsilon)-(1 / 2 \sqrt{\pi})\left|f_{0}(\epsilon)\right\rangle\left\langle f_{0}(\epsilon)\right|$. By taking only the 0 th term in the expansion of $[1-\lambda M(\epsilon)]^{-1},(12)$ reduces to

$$
\frac{\lambda}{2 \sqrt{\pi}}\left\|f_{0}(\epsilon)\right\|_{2}^{2}=\frac{\lambda}{2 \sqrt{\pi}} \int_{-\infty}^{\infty} \frac{e^{-p^{2} / 2}}{p^{2}+\epsilon^{2}} d p=1 .
$$

The latter integral has been used in relation to the calculation of the eigenvalues of the Hamiltonian of the harmonic oscillator perturbed by the rational interaction $\lambda x^{2} /\left(1+g x^{2}\right)$ (see [14-16]). Its explicit value, as a function of $\epsilon$, is

$$
\frac{\pi}{\epsilon} \exp \left(\frac{\epsilon^{2}}{2}\right) \operatorname{erfc}\left(\frac{\epsilon}{\sqrt{2}}\right) \text {. }
$$

Using such an approximation, the equation for the ground state reads

$$
\frac{\epsilon}{\lambda}=\frac{\pi}{\epsilon} \exp \left(\frac{\epsilon^{2}}{2}\right) \operatorname{erfc}\left(\frac{\epsilon}{\sqrt{2}}\right) .
$$

Using Matlab software, the above equation (15) is solved for $\epsilon_{0}(\lambda)$ for different values of $\lambda$; the ground state energy 
$E_{0}(\lambda)$ is then determined. In the case of $\lambda=0.1$, we obtain $E_{0}(0.1)=-6.89617 \times 10^{-3}$. This value is more accurate than that previously determined by taking the first two terms in the perturbation expansion of (2).

Of course, an even more accurate evaluation can be obtained by including the first order term in the series defining $[1-\lambda M(\epsilon)]^{-1}$. Then, the equation reads

$$
\frac{\lambda}{2 \sqrt{\pi}}\left\|f_{0}(\epsilon)\right\|_{2}^{2}+\frac{\lambda^{2}}{4 \pi} \sum_{n=1}^{\infty} \frac{\left(f_{0}(\epsilon), f_{2 l}(\epsilon)\right)^{2}}{2^{2 l}(2 l) !}=1 .
$$

It would be possible to write (16) almost explicitly by means of (6) and (9) in [14], where the integrals

$$
\int_{0}^{\infty} \frac{p^{2 l} \exp \left(-p^{2}\right)}{1+g p^{2}} d p
$$

are thoroughly investigated. However, we have chosen to use a slightly different strategy that enables us to improve the accuracy of our calculations without increasing heavily the mathematical complexity. Essentially, it is sufficient to take only the first two even-labeled rank-one operators in series (7) defining the trace class operator $B(\epsilon)$. As a consequence of (10), the second even rank-one operator can be written as

$$
\begin{aligned}
& \frac{1}{16 \sqrt{\pi}}\left[\alpha_{2}^{2}(\epsilon)\left|f_{0}\right\rangle\left\langle f_{0}\right|\right. \\
& \quad+\alpha_{2}(\epsilon)\left(\left|f_{0}\right\rangle\left\langle\widetilde{f_{2}}\left|+\left[\widetilde{f_{2}}\right\rangle\left\langle f_{0}\right|\right)+\mid \widetilde{f_{2}}\right\rangle\left\langle\widetilde{f_{2}}\right|\right],
\end{aligned}
$$

where we have omitted the $\epsilon$ dependence of the functions to make the notation less heavy and denoted the second summand in (10) by $\widetilde{f}_{2}$. As we have anticipated, it is reasonable to neglect the two nilpotent operators in (18) since their norm goes like $\sqrt{\epsilon}$ and, therefore, is small when $\lambda$ is small. This can be shown as follows:

$$
\frac{\alpha_{2}(\epsilon)}{16 \sqrt{\pi}} \|\left|f_{0}\right\rangle\left\langle\widetilde{f_{2}}\right|\left\|\leq \frac{\alpha_{2}(\epsilon)}{16 \sqrt{\pi}}\right\| f_{0}\left\|_{2}\right\| \widetilde{f_{2}} \|_{2} .
$$

Using the explicit formulae for $\alpha_{2}(\epsilon)$ and $\widetilde{f_{2}}$, the latter becomes

$$
\begin{aligned}
& \frac{1}{16 \sqrt{\pi}}\left[\frac{(2 \pi)^{1 / 2}}{\left\|f_{0}\right\|_{2}}-\epsilon^{2}\left\|f_{0}\right\|_{2}\right]\left\|\widetilde{f}_{2}\right\|_{2} \\
& =\frac{\sqrt{\epsilon}}{16 \sqrt{\pi}}\left[\frac{\sqrt{2} \exp \left(-\epsilon^{2} / 4\right)}{\sqrt{\operatorname{erfc}(\epsilon / \sqrt{2})}}\right. \\
& \left.-\epsilon \sqrt{\pi} \exp \left(\frac{\epsilon^{2}}{4}\right) \sqrt{\operatorname{erfc}\left(\frac{\epsilon}{\sqrt{2}}\right)}\right]\left[\sqrt{\pi}\left(1+\sqrt{2} \epsilon^{2}\right)\right. \\
& \left.-\frac{2 \epsilon \exp \left(-\epsilon^{2} / 2\right)}{\operatorname{erfc}(\epsilon / \sqrt{2})}\right]^{1 / 2}
\end{aligned}
$$

which proves our claim. Of course, the norm of $\left|\widetilde{f_{2}}\right\rangle\left\langle\widetilde{f_{2}}\right|$, namely, the quantity inside the square root in the last factor

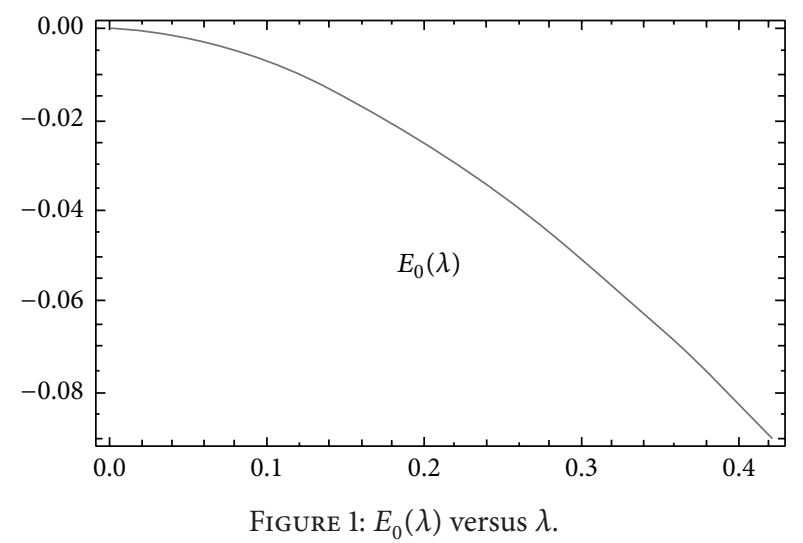

TABLE 1: The ground state energy for various values of the coupling constant.

\begin{tabular}{lc}
\hline$\lambda$ & $E_{0}(\lambda)$ \\
\hline 0.01 & $-7.7447596 \times 10^{-5}$ \\
0.05 & $-1.835472435 \times 10^{-3}$ \\
0.1 & $-6.902786581 \times 10^{-3}$ \\
0.15 & $-1.467219033 \times 10^{-3}$ \\
0.2 & $-2.474052522 \times 10^{-2}$ \\
\hline
\end{tabular}

of (20), is not small in $\epsilon$ but becomes small because of the smallness of the coupling constant by which it must be multiplied. Therefore, the second symmetric eigenstate can only be created when the coupling constant exceeds a certain threshold.

As a consequence of our estimates, the more accurate transcendental equation is obtained by simply taking account of the term involving $\alpha_{2}^{2}(\epsilon)$. Although the algebra is a bit lengthy, there is no difficulty in the determination of the new transcendental equation required. We give its final form omitting the intermediate steps:

$$
\begin{aligned}
& \epsilon\left(\frac{1}{\lambda}+\frac{\epsilon^{2}}{2^{5 / 2}}\right) \\
& \quad=\frac{\sqrt{\pi}}{2}\left[\left(1+\frac{\epsilon^{4}}{8}\right) \exp \left(\frac{\epsilon^{2}}{2}\right) \operatorname{erfc}\left(\frac{\epsilon}{\sqrt{2}}\right)\right. \\
& \left.+\frac{\epsilon^{2} \exp \left(-\epsilon^{2} / 2\right)}{4 \pi \operatorname{erfc}(\epsilon / \sqrt{2})}\right] .
\end{aligned}
$$

Solving the above equation using Matlab for various small values of $\lambda$, for which the truncation is satisfactory, leads to the values of $E_{0}(\lambda)$ shown in Figure 1 (with some particular values singled out in Table 1 ) for each value of $\lambda$ in the interval $[0,0.2]$.

Using a Sleign2 algorithm (see [17]) for the calculation of eigenvalues of one-dimensional Schrödinger operators for the case of $\lambda=0.1$, a value of $E_{0}(0.1)=-0.006903033148$ is determined; the method developed in this paper produces a value of $E_{0}(0.1)=-0.006902786581$ which is a fairly good agreement. 
Remark. Since the solution $\epsilon_{0}(\lambda)$ of (21) is near zero, where both the Gaussian and the complementary error function in the last term of the right hand side of the equation are close to one, such a term does not represent a problem in the numerical computation using Matlab. However, if one is interested in the solution for greater values of $\lambda$, it is advisable to recast (18) into a slightly different form eliminating the ratio $\exp \left(-\epsilon^{2} / 2\right) / \operatorname{erfc}(\epsilon / \sqrt{2})$, which does become of the type $0 / 0$, numerically speaking, very quickly due to the fast decay of both functions.

The results presented in both Table 1 and Figure 1 complete the analysis of this paper. The natural continuation of this work would be to investigate the spectroscopy for the one-dimensional Hamiltonian for greater values of $\lambda$, for which additional bound states appear. Furthermore, we should use the same technique to compute the ground state in two dimensions, even though we expect to deal with slightly different integrals. Our preliminary calculations lead us to believe that our technique should work also for the case of an anisotropic Gaussian well. As is well known, the three-dimensional situation is quite different since we have the existence of a coupling constant threshold even for the ground state energy. Nevertheless, we believe we should be able to determine such a threshold and the ground state energy also in that case.

\section{Conflict of Interests}

The authors declare that there is no conflict of interests regarding the publication of this paper.

\section{Acknowledgments}

The authors would like to thank Professor Martin Klaus (Virginia Tech) and S. Manservisi for several useful discussions at the time when this paper was conceived. G. Muchatibaya and J. Mushanyu would like to thank the support of the University of Zimbabwe when this project was underway. S. Fassari and F. Rinaldi kindly acknowledge the constant encouragement and support granted to them by the Director of CERFIM, Professor D. Merlini.

\section{References}

[1] R. C. Ashoori, "Electrons in artificial atoms," Nature, vol. 379, no. 6564, pp. 413-419, 1996.

[2] S. Fassari and F. Rinaldi, "On the spectrum of the Schrödinger Hamiltonian with a particular configuration of three onedimensional point interactions," Reports on Mathematical Physics, vol. 64, no. 3, pp. 367-393, 2009.

[3] M. Klaus, "Some applications of the Birman-Schwinger principle," Helvetica Physica Acta, vol. 55, pp. 413-419, 1982.

[4] M. Klaus, "On the bound state of Schrödinger operators in one dimension," Annals of Physics, vol. 108, no. 2, pp. 288-300, 1977.

[5] S. Nandi, "The quantum Gaussian well," American Journal of Physics, vol. 78, no. 12, pp. 1341-1345, 2010.
[6] B. Simon, "The bound state of weakly coupled Schrödinger operators in one and two dimensions," Annals of Physics, vol. 97, no. 2, pp. 279-288, 1976.

[7] F. M. Fernández, "Quantum Gaussian wells and barriers," American Journal of Physics, vol. 79, no. 7, pp. 752-754, 2011.

[8] F. M. Fernández, "Simple variationalapproaches to quantum wells," 2012, http://arxiv.org/pdf/1204.0783.pdf.

[9] P. B. Bailey, W. N. Everitt, and A. Zettl, "Computing eigenvalues of singular Sturm-Liouville problems," Results in Mathematics, vol. 20, no. 1-2, pp. 391-423, 1991.

[10] E. Brändas, M. Rittby, and N. Elander, "Titchmarsh-Weyl theory and its relations to scattering theory: spectral densities and cross sections; theory and applications," Journal of Mathematical Physics, vol. 26, article 2648, 1985.

[11] W. N. Everitt and A. G. Halvorsen, "On the asymptotic form of the Titchmarsh-Weyl m-coefficient," Applicable Analysis, vol. 8, pp. 153-169, 1978.

[12] S. Fassari, "An estimate regarding one-dimensional point interactions," Helvetica Physica Acta, vol. 68, no. 2, pp. 121-125, 1995.

[13] M. Reed and B. Simon, Scattering Theory, Academic Press, New York, NY, USA, 1979.

[14] N. Bessis and G. Bessis, "A note on the Schrödinger equation for the $x^{2}+\lambda x^{2} /\left(1+g x^{2}\right)$ potential," Journal of Mathematical Physics, vol. 21, no. 12, pp. 2780-2785, 1980.

[15] S. Fassari, "A note on the eigenvalues of the Hamiltonian of the harmonic oscillator perturbed by the potential $\lambda x^{2} /\left(1+g x^{2}\right)$," Reports on Mathematical Physics, vol. 37, no. 2, pp. 283-293, 1996.

[16] A. K. Mitra, "On the interaction of the type $x^{2}+\lambda x^{2} /\left(1+g x^{2}\right)$," Journal of Mathematical Physics, vol. 19, no. 10, pp. 2018-2022, 1978.

[17] P. B. Bailey, W. N. Everitt, and A. Zettl, "The SLEIGN2 SturmLiouville code," ACM Transactions on Mathematical Software, vol. 27, pp. 143-192, 2001. 


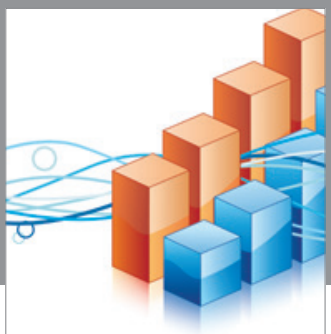

Advances in

Operations Research

vatem alat4

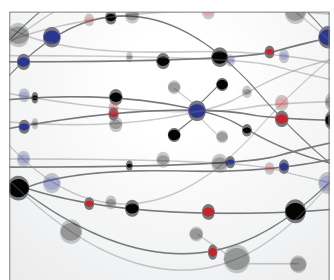

\section{The Scientific} World Journal
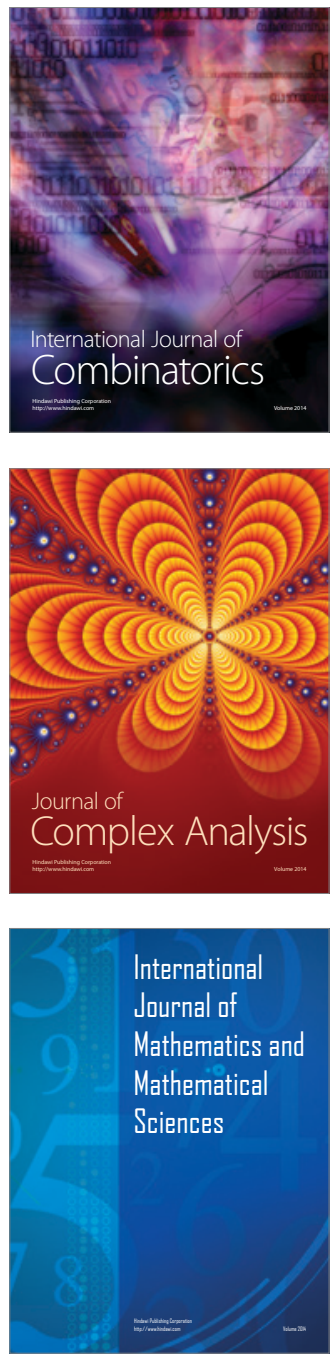
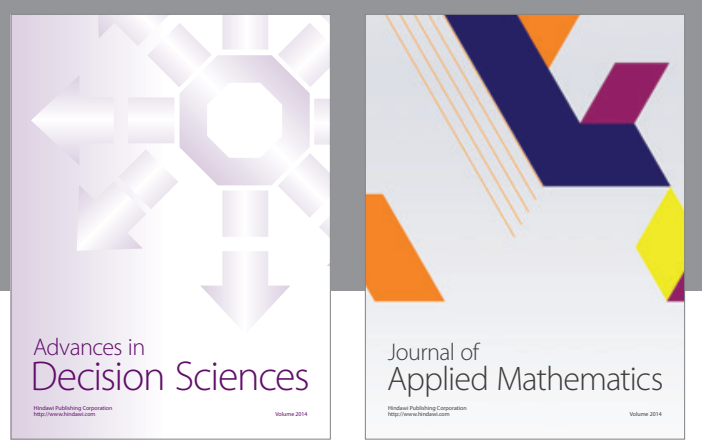

Algebra

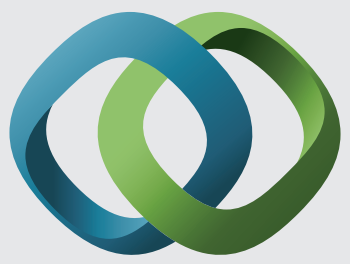

\section{Hindawi}

Submit your manuscripts at

http://www.hindawi.com
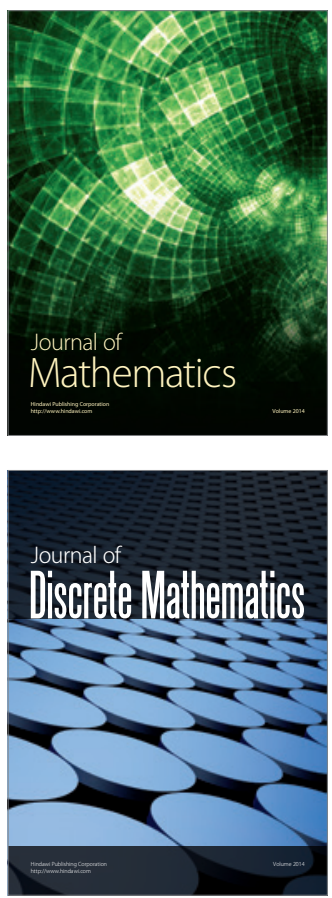

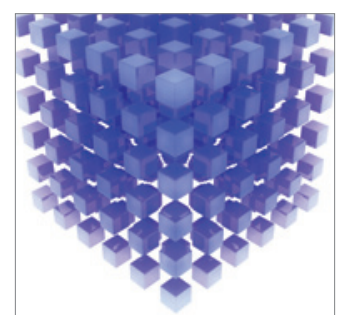

Mathematical Problems in Engineering
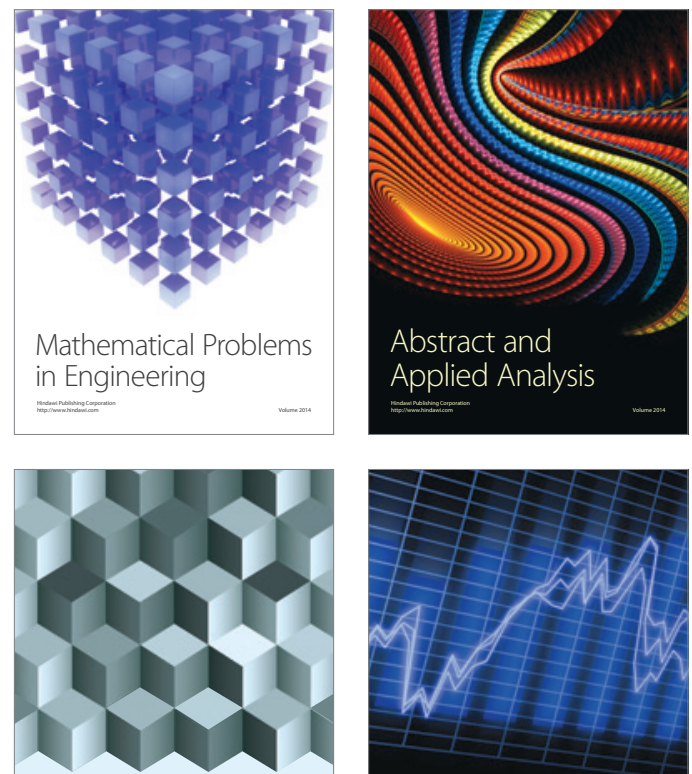

Journal of

Function Spaces

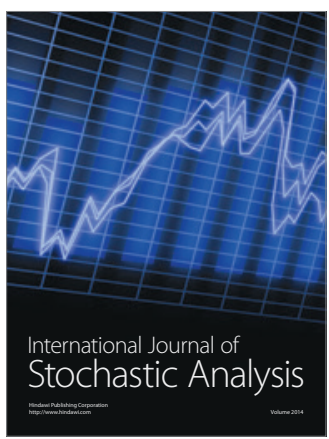

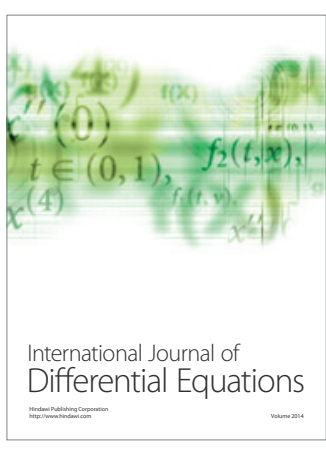
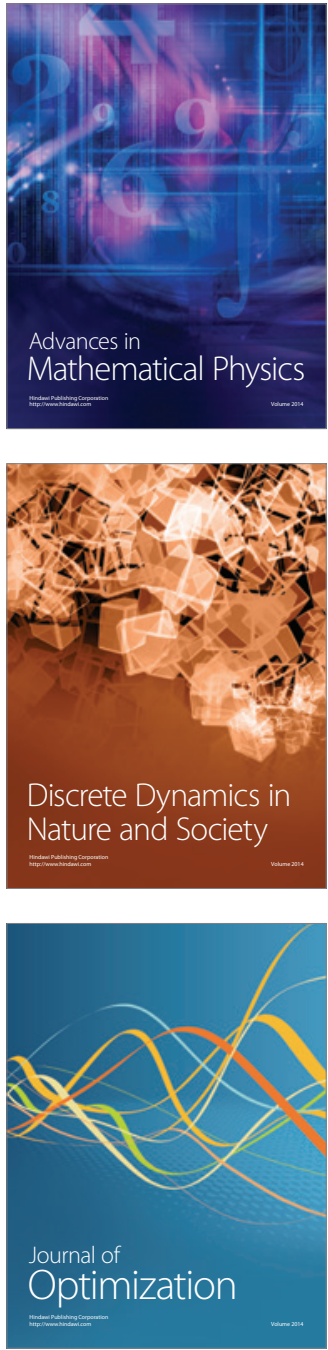